\title{
Fixed Point Theorems on Nonlinear Binary Operator Equations with Applications
}

\author{
Baomin Qiao \\ Department of Mathematics, Shangqiu Normal College, Shangqiu 476000, China \\ Correspondence should be addressed to Baomin Qiao; bmqiao@126.com
}

Received 18 April 2014; Revised 11 June 2014; Accepted 11 June 2014; Published 19 June 2014

Academic Editor: Krzysztof Ciepliński

Copyright (C) 2014 Baomin Qiao. This is an open access article distributed under the Creative Commons Attribution License, which permits unrestricted use, distribution, and reproduction in any medium, provided the original work is properly cited.

The existence and uniqueness for solution of systems of some binary nonlinear operator equations are discussed by using cone and partial order theory and monotone iteration theory. Furthermore, error estimates for iterative sequences and some corresponding results are obtained. Finally, the applications of our results are given.

\section{Introduction}

In recent years, more and more scholars have studied binary operator equations and have obtained many conclusions; see [1-6]. In this paper, we will discuss solutions for these equations which associated with an ordinal symmetric contraction operator and obtain some results which generalized and improved those of [3-6]. Finally, we apply our conclusions to two-point boundary value problem with two-degree superlinear ordinary differential equations.

In the following, let $E$ always be a real Banach space which is partially ordered by a cone $P$, let $P$ be a normal cone of $E$, $N$ is normal constant of $P$, partial order $\leq$ is determined by $P$ and $\theta$ denotes zero element of $E$. Let $u, v \in E, u<v, D=$ $[u, v]=\{x \in E: u \leq x \leq v\}$ denote an ordering interval of $E$.

For the concepts of normal cone and partially order, mixed monotone operator, coupled solutions of operator equations, and so forth see $[1,5]$.

Definition 1. Let $A: D \times D \rightarrow E$ be a binary operator. $A$ is said to be $L$-ordering symmetric contraction operator if there exists a bounded linear and positive operator $L: E \rightarrow E$, where spectral radius $r(L)<1$ such that $A(y, x)-A(x, y) \leq$ $L(y-x)$ for any $x, y \in D, x \leq y$, where $L$ is called a contraction operator of $A$.

\section{Main Results}

Theorem 2. Let $A: D \times D \rightarrow E$ be L-ordering symmetric contraction operator, and there exists a $\alpha \in[0,1)$ such that

$$
\begin{gathered}
A\left(x_{2}, y_{2}\right)-A\left(x_{1}, y_{1}\right) \geq-\alpha\left(x_{2}-x_{1}\right), \\
u \leq x_{1} \leq x_{2} \leq v, u \leq y_{2} \leq y_{1} \leq v .
\end{gathered}
$$

If condition $\left(H_{1}\right) u \leq A(u, v), A(v, u) \leq v-\alpha(v-u)$ or $\left(H_{2}\right)$ $u+\alpha(v-u) \leq A(u, v), A(v, u) \leq v$ holds, then the following statements hold.

$\left(C_{1}\right) A(x, x)=x$ has a unique solution $x^{*} \in D$, and for any coupled solutions $x, y \in D, x=y=x^{*}$.

$\left(C_{2}\right)$ For any $x_{0}, y_{0} \in D$, we construct symmetric iterative sequences:

$$
\begin{array}{r}
x_{n}=\frac{1}{\alpha+1}\left[A\left(x_{n-1}, y_{n-1}\right)+\alpha x_{n-1}\right], \\
y_{n}=\frac{1}{\alpha+1}\left[A\left(y_{n-1}, x_{n-1}\right)+\alpha y_{n-1}\right], \\
n=1,2,3, \ldots .
\end{array}
$$


Then $x_{n} \rightarrow x^{*}, y_{n} \rightarrow x^{*}(n \rightarrow \infty)$, and for any $\beta \in$ $(r(L), 1)$, there exists a natural number $m$; and if $n \geq m$, we get error estimates for iterative sequences (2):

$$
\left\|x_{n}\left(y_{n}\right)-x^{*}\right\| \leq 2 N\left(\frac{\alpha+\beta}{\alpha+1}\right)^{n}\|u-v\| .
$$

Proof. Set $B(x, y)=(1 /(\alpha+1))[A(x, y)+\alpha x]$, and if condition $\left(\mathrm{H}_{1}\right)$ or $\left(\mathrm{H}_{2}\right)$ holds, then it is obvious that

$$
u \leq B(u, v), \quad B(v, u) \leq v .
$$

By (1), we easily prove that $B: D \times D \rightarrow E$ is mixed monotone operator, and for any $x, y \in D, u \leq x \leq y \leq v$,

$$
\theta \leq B(y, x)-B(x, y) \leq H(y-x),
$$

where $H=(1 /(\alpha+1))(L+\alpha I)$ is a bounded linear and positive operator and $I$, is identical operator.

By the mathematical induction, we easily prove that

$$
\theta \leq B^{n}(y, x)-B^{n}(x, y) \leq H^{n}(y-x), \quad u \leq x \leq y \leq v,
$$

where $B^{n}(x, y)=B\left(B^{n-1}(x, y), B^{n-1}(y, x)\right), x, y \in D, n \geq 2$. By the character of normal cone $P$, it is shown that

$$
\left\|B^{n}(y, x)-B^{n}(x, y)\right\| \leq N\left\|H^{n}\right\|\|y-x\|, \quad u \leq x \leq y \leq v .
$$

For any $\beta \in(r(L), 1)$, since $\lim _{n \rightarrow \infty}\left\|H^{n}\right\|^{1 / n}=r(H) \leq$ $(\alpha+r(L)) /(\alpha+1)<(\alpha+\beta) /(\alpha+1)<1$, there exists a natural number $m$, and if $n \geq m$, we have $\left\|H^{n}\right\|<((\alpha+\beta) /(\alpha+1))^{n}$, and $N\left\|H^{m}\right\|<1$. Considering mixed monotone operator $B^{m}$ and constant $N\left\|H^{m}\right\|, B^{m}(x, x)=x$ has a unique solution $x^{*}$ and for any coupled solution $x, y \in D$, such that $x=y=x^{*}$ by Theorem 3 in [3].

From $B^{m}\left(B\left(x^{*}, x^{*}\right), B\left(x^{*}, x^{*}\right)\right)=B\left(B^{m}\left(x^{*}, x^{*}\right), B^{m}\left(x^{*}\right.\right.$, $\left.\left.x^{*}\right)\right)=B\left(x^{*}, x^{*}\right)$, and the uniqueness of solution with $B^{m}(x, x)=x$, then we have $B\left(x^{*}, x^{*}\right)=x^{*}$ and $A\left(x^{*}, x^{*}\right)=$ $x^{*}$.

We take note of that $A(x, x)=x$ and $B(x, x)=x$ have the same coupled solution; therefore, a coupled solution for $B(x, x)=x$ must be a coupled solution for $B^{m}(x, x)=x$; consequently, $\left(\mathrm{C}_{1}\right)$ has been proved.

Considering iterative sequence (2), we construct iterative sequences:

$$
u_{n}=B\left(u_{n-1}, v_{n-1}\right), \quad v_{n}=B\left(v_{n-1}, u_{n-1}\right),
$$

where $u_{0}=u, v_{0}=v$, it is obvious that

$$
\begin{gathered}
x_{n}=B\left(x_{n-1}, y_{n-1}\right), \quad y_{n}=B\left(y_{n-1}, x_{n-1}\right), \\
\theta \leq v_{n}-u_{n} \leq H^{n}(v-u),
\end{gathered}
$$

by the mathematical induction and characterization of mixed monotone of $B$; then

$$
u_{n} \leq x^{*} \leq v_{n}, \quad u_{n} \leq x_{n} \leq v_{n}, \quad u_{n} \leq y_{n} \leq v_{n} .
$$

Hence,

$$
\begin{array}{r}
\left\|x_{n}\left(y_{n}\right)-u_{n}\right\| \leq N\left\|v_{n}-u_{n}\right\|, \\
\left\|x^{*}-u_{n}\right\| \leq N\left\|v_{n}-u_{n}\right\|, \\
n=1,2,3, \ldots
\end{array}
$$

Moreover, if $n \geq m$, we get

$$
\begin{aligned}
& \left\|x_{n}\left(y_{n}\right)-x^{*}\right\| \leq 2 N\left\|v_{n}-u_{n}\right\| \\
& \quad \leq 2 N\left\|H^{n}\right\|\|v-u\| \leq 2 N\left(\frac{\alpha+\beta}{\alpha+1}\right)^{n}\|u-v\| .
\end{aligned}
$$

Consequently, $x_{n} \rightarrow x^{*}, y_{n} \rightarrow x^{*}(n \rightarrow \infty)$.

Remark 3. When $\alpha=0$, Theorem 1 in [4] is a special case of this paper Theorem 2 under condition $\left(\mathrm{H}_{1}\right)$ or $\left(\mathrm{H}_{2}\right)$.

Corollary 4. Let $A: D \times D \rightarrow E$ be L-ordering symmetric contraction operator; if there exists a $\alpha \in[0,1)$ such that $A$ satisfies condition of Theorem 2, the following statement holds.

$\left(C_{3}\right)$ For any $\beta \in(r(L), 1)$ and $\alpha+\beta<1$, we make iterative sequences:

$$
\begin{aligned}
& u_{n}=A\left(u_{n-1}, v_{n-1}\right), \\
& v_{n}=A\left(v_{n-1}, u_{n-1}\right)+\alpha\left(v_{n-1}-u_{n-1}\right), \\
& n=1,2,3, \ldots,
\end{aligned}
$$

or

$$
\begin{aligned}
& u_{n}=A\left(u_{n-1}, v_{n-1}\right)-\alpha\left(v_{n-1}-u_{n-1}\right), \\
& v_{n}=A\left(v_{n-1}, u_{n-1}\right),
\end{aligned}
$$

$$
n=1,2,3, \ldots,
$$

where $u_{0}=u, v_{0}=v$.

Thus, $u_{n} \rightarrow x^{*}, v_{n} \rightarrow x^{*}(n \rightarrow \infty)$, and there exists a natural number $m$, and if $n \geq m$, we have error estimates for iterative sequences (13) or (14):

$$
\left\|u_{n}\left(v_{n}\right)-x^{*}\right\| \leq N(\alpha+\beta)^{n}\|u-v\| .
$$

Proof. By the character of mixed monotone of $A$, then (1) and $\left(\mathrm{C}_{1}\right),\left(\mathrm{C}_{2}\right)$ [in $(1),\left(\mathrm{C}_{2}\right)$ where $\alpha=0$ ] hold.

In the following, we will prove $\left(\mathrm{C}_{3}\right)$.

Consider iterative sequence (13); since $u \leq x^{*} \leq v$, we get

$$
\begin{aligned}
u_{1} & =A(u, v) \leq A\left(x^{*}, x^{*}\right)=x^{*} \leq A(v, u) \\
& =v_{1}-\alpha(v-u) \leq v_{1} .
\end{aligned}
$$

By the mathematical induction, we easily prove $u_{n} \leq x^{*} \leq v_{n}$, $n \geq 1$, hence

$$
\theta \leq x^{*}-u_{n} \leq v_{n}-u_{n}, \quad \theta \leq v_{n}-x^{*} \leq v_{n}-u_{n} .
$$

It is clear that

$$
\begin{aligned}
\theta & \leq v_{n}-u_{n} \leq(L+\alpha I)\left(v_{n-1}-u_{n-1}\right) \\
& =(L+\alpha I)^{n}(v-u), \quad n \geq 1 .
\end{aligned}
$$




$$
\begin{aligned}
& \text { For any } \beta \in(r(L), 1), \alpha+\beta<1 \text {, since } \\
& \lim _{n \rightarrow \infty}\left\|(L+\alpha I)^{n}\right\|^{1 / n}=r(L+\alpha I) \leq r(L)+\alpha<\alpha+\beta<1,
\end{aligned}
$$

there exists a natural number $m$, if $n \geq m$, such that

$$
\left\|(L+\alpha I)^{n}\right\|<(\alpha+\beta)^{n} .
$$

Moreover,

$$
\begin{gathered}
\left\|u_{n}\left(v_{n}\right)-x^{*}\right\| \leq N\left\|(L+\alpha I)^{n}\right\|\|u-v\| \\
\leq N(\alpha+\beta)^{n}\|u-v\|, \quad(n \geq m) .
\end{gathered}
$$

Consequently, $u_{n} \rightarrow x^{*}, v_{n} \rightarrow x^{*},(n \rightarrow \infty)$.

Similarly, we can prove (14).

Theorem 5. Let $A: D \times D \rightarrow E$ be a L-ordering symmetric contraction operator; if there exists a $\alpha \in[0,1)$ such that $(1-$ $\alpha) u \leq A(u, v), A(v, u) \leq(1-\alpha) v$, then the following statements hold.

$\left(C_{4}\right)$ Operator equation $A(x, x)=(1-\alpha) x$ has a unique solution $x^{*} \in D$, and for any coupled solutions $x, y \in D, x=$ $y=x^{*}$.

$\left(C_{5}\right)$ For any $x_{0}, y_{0}, w_{0}, z_{0} \in D$, we make symmetric iterative sequences

$$
\begin{array}{r}
x_{n}=\frac{1}{1-\alpha} A\left(x_{n-1}, y_{n-1}\right), \\
y_{n}=\frac{1}{1-\alpha} A\left(y_{n-1}, x_{n-1}\right), \\
n=1,2,3, \ldots, \\
w_{n}=A\left(w_{n-1}, z_{n-1}\right)+\alpha w_{n-1}, \\
z_{n}=A\left(z_{n-1}, w_{n-1}\right)+\alpha z_{n-1}, \\
n=1,2,3, \ldots
\end{array}
$$

Then $x_{n} \rightarrow x^{*}, y_{n} \rightarrow x^{*}, w_{n} \rightarrow x^{*}, z_{n} \rightarrow x^{*}(n \rightarrow \infty)$, and for any $\beta \in(r(L), 1), \alpha+\beta<1$, there exists a natural number $m$, and if $n \geq m$, then we have error estimates for iterative sequences (22) and (23), respectively,

$$
\begin{aligned}
& \left\|x_{n}\left(y_{n}\right)-x^{*}\right\| \leq 2 N\left(\frac{\beta}{1-\alpha}\right)^{n}\|u-v\|, \\
& \left\|w_{n}\left(z_{n}\right)-x^{*}\right\| \leq 2 N(\alpha+\beta)^{n}\|u-v\| .
\end{aligned}
$$

Proof. Set $B(x, y)=(1 /(1-\alpha)) A(x, y)$ or $C(x, y)=$ $A(x, y)+\alpha x$; we can prove that this theorem imitates proof of Theorem 2.

Similarly, we can prove the following theorems.

Theorem 6. Let $A: D \times D \rightarrow E$ be L-ordering symmetric contraction operator; if there exists a $\alpha \in[0,1)$ such that $u+$ $\alpha v \leq A(u, v), A(v, u) \leq v+\alpha u$, then the following statements hold.
$\left(C_{6}\right)$ Equation $A(x, x)=(1+\alpha) x$ has a unique solution $x^{*} \in D$, and for any coupled solutions $x, y \in D x=y=x^{*}$.

$\left(C_{7}\right)$ For any $x_{0}, y_{0} \in D$, we make symmetric iterative sequence:

$$
\begin{array}{r}
x_{n}=\frac{1}{1+\alpha} A\left(x_{n-1}, y_{n-1}\right), \\
y_{n}=\frac{1}{1+\alpha} A\left(y_{n-1}, x_{n-1}\right), \\
n=1,2,3, \ldots .
\end{array}
$$

Then $x_{n} \rightarrow x^{*}, y_{n} \rightarrow x^{*}(n \rightarrow \infty)$; moreover, $\beta \in(r(L), 1)$, and there exists natural number $m$, and if $n \geq m$, then we have error estimates for iterative sequence (25):

$$
\left\|x_{n}\left(y_{n}\right)-x^{*}\right\| \leq 2 N\left(\frac{\beta}{\alpha+1}\right)^{n}\|u-v\|,
$$

$\left(C_{8}\right)$ For any $\beta \in(r(L), 1)(\alpha+\beta<1), w_{0}, z_{0} \in D$, we make symmetry iterative sequence $w_{n}=A\left(w_{n-1}, z_{n-1}\right)-\alpha z_{n-1}, z_{n}=$ $A\left(z_{n-1}, w_{n-1}\right)-\alpha w_{n-1}, n \geq 1$; then $w_{n} \rightarrow x^{*}, z_{n} \rightarrow$ $x^{*}(n \rightarrow \infty)$, and there exists a natural number $m$, and if $n \geq m$, we have error estimates for iterative sequence (24).

Remark 7. When $\alpha=0$, Corollary 2 in [4] is a special case of this paper Theorems 2-6.

Remark 8. The contraction constant of operator in [5] is expand into the contraction operator of this paper.

Remark 9. Operator $A$ of this paper does not need character of mixed monotone as operator in [6].

\section{Application}

We consider that two-point boundary value problem for twodegree super linear ordinary differential equations:

$$
\begin{gathered}
x^{\prime \prime}+a(t) x^{m}+\frac{1}{1+b(t) x}=0, \quad t \in[0,1],(m \geq 2) \\
x(0)=x^{\prime}(1)=0 .
\end{gathered}
$$

Let $k(t, s)$ be Green function with boundary value problem (23); that is,

$$
k(t, s)=\min \{t, s\}= \begin{cases}t, & t \leq s \\ s, & s<t .\end{cases}
$$

Then the solution with boundary value problem (23) and solution for nonlinear integral equation with type of Hammerstein

$$
x(t)=\int_{0}^{1} k(t, s)\left\{a(s)[x(s)]^{m}+\frac{1}{1+b(s) x(s)}\right\} d s
$$

are equivalent, where $\max _{t \in[0,1]} \int_{0}^{1} k(t, s) d s=1 / 2$.

Theorem 10. Let $a(t), b(t)$ be nonnegative continuous function in $[0,1], p=\max _{t \in[0,1]} a(t), q=\max _{t \in[0,1]} b(t)$. If $p<1, m p+$ 
$q<2$, then boundary value problem (23) has a unique solution $x^{*}(t)$ such that $0 \leq x^{*}(t) \leq 1(t \in[0,1])$. Moreover, for any initial function $x_{0}(t), y_{0}(t)$, such that

$$
0 \leq x_{0}(t) \leq 1, \quad 0 \leq y_{0}(t) \leq 1 \quad(t \in[0,1])
$$

we make iterative sequence:

$$
\begin{gathered}
x_{n}(t)=\int_{0}^{1} k(t, s)\left\{a(s)\left[x_{n-1}(s)\right]^{m}+\frac{1}{1+b(s) y_{n-1}(s)}\right\} d s, \\
y_{n}(t)=\int_{0}^{1} k(t, s)\left\{a(s)\left[y_{n-1}(s)\right]^{m}+\frac{1}{1+b(s) x_{n-1}(s)}\right\} d s, \\
n=1,2,3, \ldots
\end{gathered}
$$

Then $x_{n}(t)$ and $y_{n}(t)$ are all uniformly converge to $x^{*}(t)$ on $[0,1]$, and we have error estimates:

$$
\begin{array}{r}
\left|x_{n}(t)\left(y_{n}(t)\right)-x^{*}(t)\right| \leq 2\left(\frac{m p+q}{2}\right)^{n}, \\
t \in[0,1], n=1,2,3, \ldots
\end{array}
$$

Proof. Let $E=C[0,1], P=\{x \in E \mid x(t) \geq 0, t \in$ $[0,1]\},\|x\|=\max _{t \in[0,1]}|x(t)|$ denote norm of; then $E$ has become Banach space, $P$ is normal cone of $E$, and its normal constant $N=1$. It is obvious that integral Equation (24) transforms to operator equation $A(x, x)=x$, where

$$
\begin{aligned}
& A(x, y)(t) \\
& \quad=\int_{0}^{1} k(t, s)\left\{a(s)[x(s)]^{m}+\frac{1}{1+b(s) y(s)}\right\} d s, \quad t \in[0,1] .
\end{aligned}
$$

Set $u=u(t) \equiv 0, v=v(t) \equiv 1$; then $D=[0,1]$ denote ordering interval of $E, A: D \times D \rightarrow E$ is mixed monotone operator, and $0 \leq A(0,1), A(1,0) \leq(1+p) / 2<1$.

Set

$$
L x(t)=\int_{0}^{1} k(s, t)[m a(s)+b(s)] x(s) d s, \quad t \in[0,1] .
$$

Then $L: E \rightarrow E$ is bounded linear operator, its spectral radius $r(L) \leq(m p+q) / 2<1$, and for any $x, y \in E, 0 \leq x(t) \leq$ $y(t) \leq 1$ such that $0 \leq A(y, x)(t)-A(x, y)(t) \leq L(y-x)(t), A$ is $L$-ordering symmetric contraction operator, by Theorem 2 (where $\alpha=0$ ); then Theorem 10 has been proved.

\section{Conflict of Interests}

The author declares that there is no conflict of interests regarding the publication of this paper.

\section{Acknowledgments}

This work is supported by the NSF of Henan Education Bureau (2000110019) and by the NSF of Shangqiu (200211125).

\section{References}

[1] D. J. Guo and V. Lakshmikantham, "Coupled fixed points of nonlinear operators with applications," Nonlinear Analysis: Theory, Methods \& Applications, vol. 11, no. 5, pp. 623-632, 1987.

[2] Y. Sun, "A fixed point theorem for mixed monotone operators with applications," Journal of Mathematical Analysis and Applications, vol. 156, no. 1, pp. 240-252, 1991.

[3] Q. Zhang, "Contraction mapping principle of mixed monotone mapping and applications," Henan Science, vol. 18, no. 2, pp. 121$125,2000$.

[4] J. X. Sun and L. S. Liu, "An iterative solution method for nonlinear operator equations and its applications," Acta Mathematica Scientia A, vol. 13, no. 2, pp. 141-145, 1993.

[5] Q. Zhang, "Iterative solutions of ordering symmetric contraction operator with applications," Journal of Engineering Mathematics, vol. 17, no. 2, pp. 131-134, 2000.

[6] X. L. Yan, "Fixed-point theorems for mixed monotone operators and their applications," Mathematica Applicata, vol. 4, no. 4, pp. 107-114, 1991. 


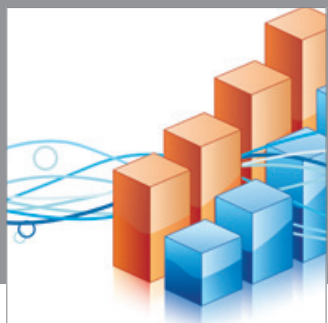

Advances in

Operations Research

mansans

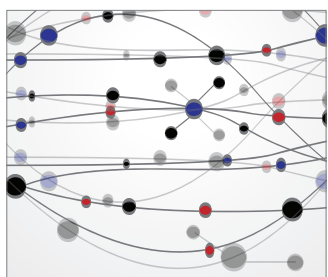

The Scientific World Journal
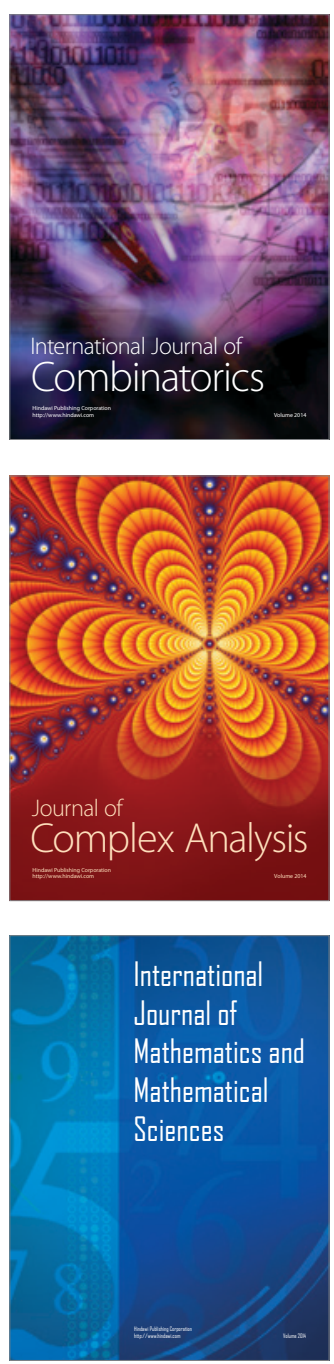
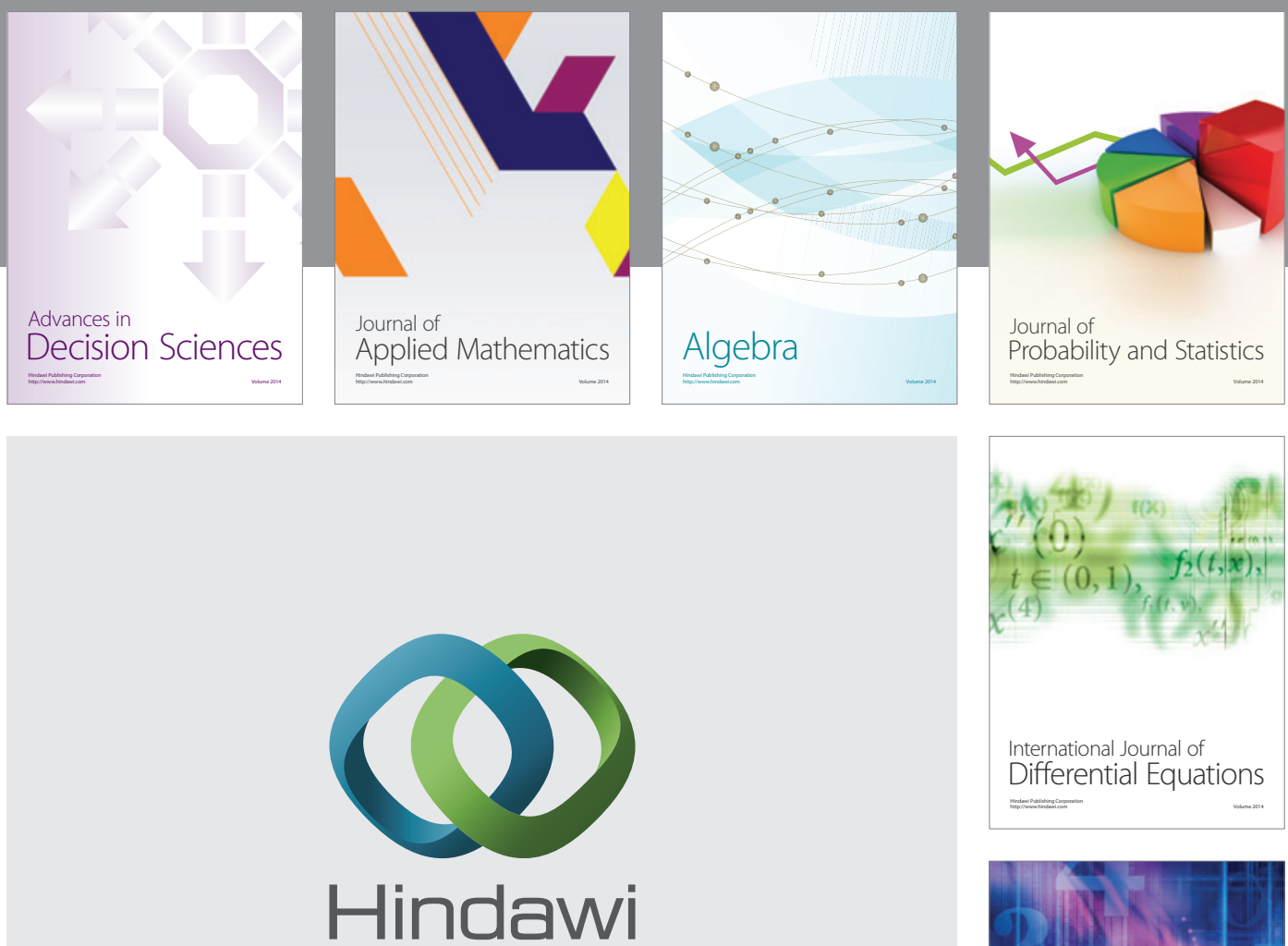

Submit your manuscripts at http://www.hindawi.com
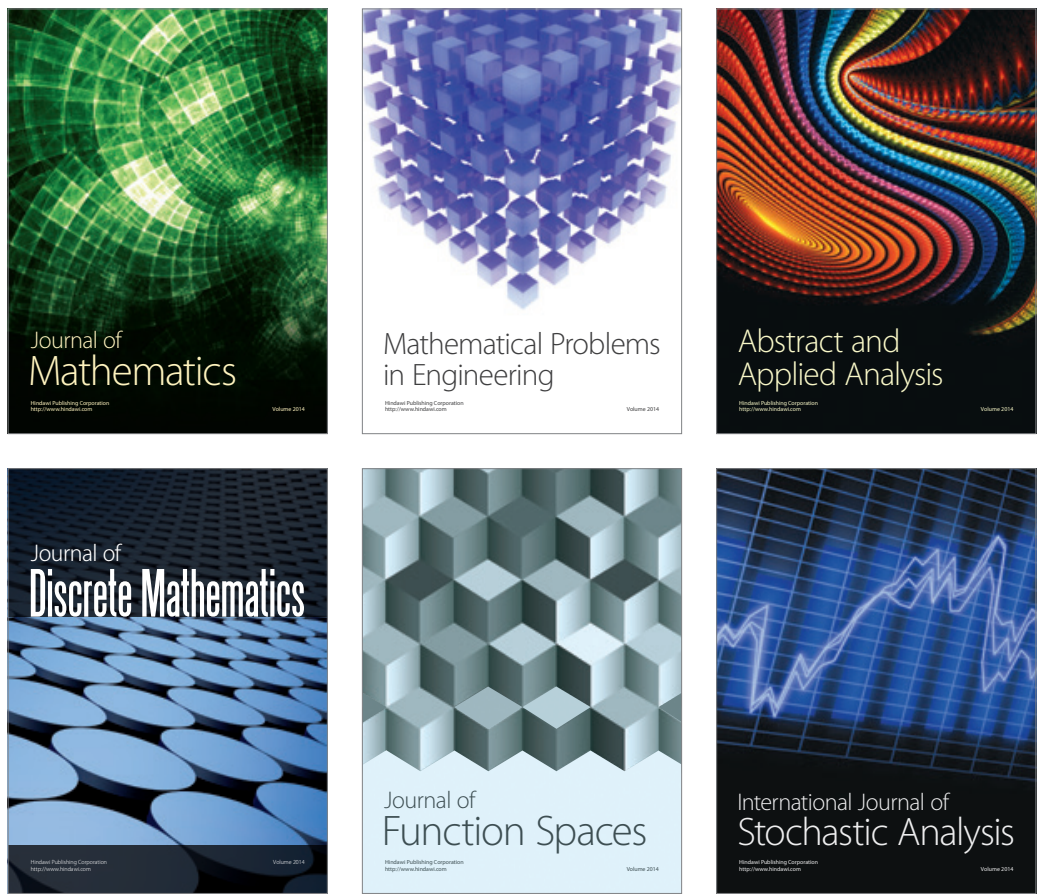

Journal of

Function Spaces

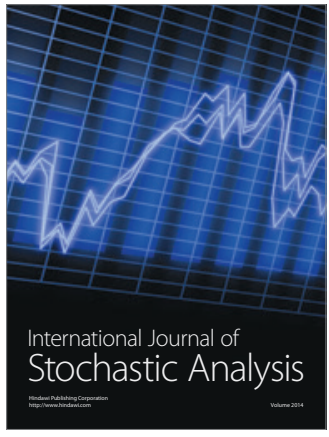

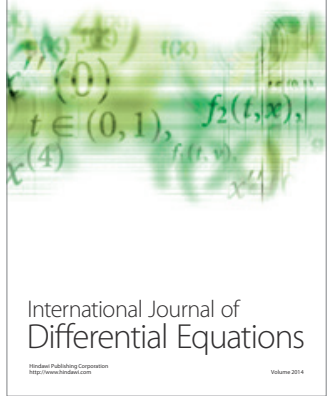
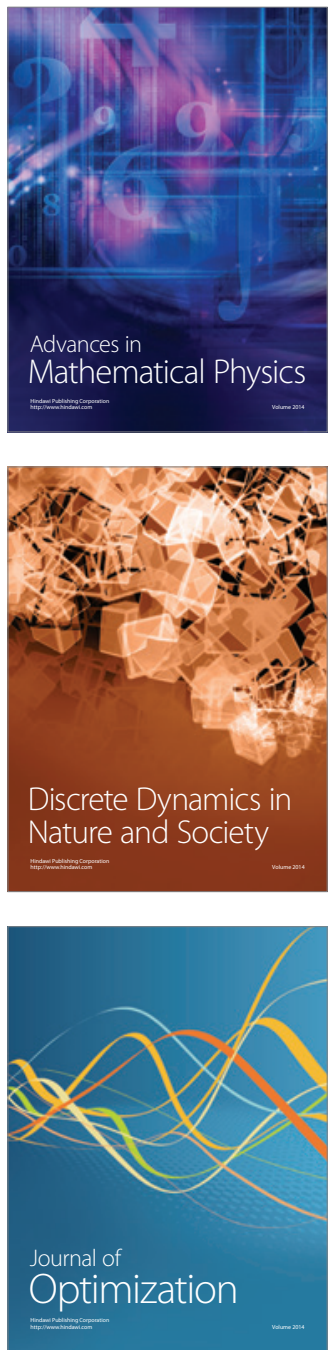